\title{
Positioning errors of dental implants and their associations with adjacent structures and anatomical variations: A CBCT-based study
}

\author{
Beatriz Ribeiro Ribas $\mathbb{D}^{1}$, Eduarda Helena Leandro Nascimento(D) ${ }^{1 *}$, Deborah Queiroz Freitas (D)2,

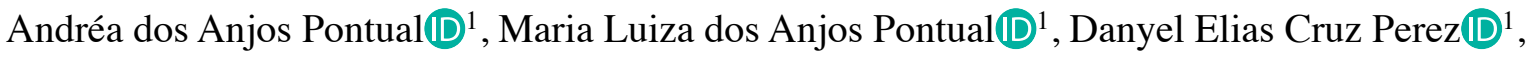 \\ Flávia Maria Moraes Ramos-Perez(D) ${ }^{1}$ \\ ${ }^{1}$ Department of Clinical and Preventive Dentistry, Federal University of Pernambuco, Recife, Pernambuco, Brazil \\ ${ }^{2}$ Division of Oral Radiology, Department of Oral Diagnosis, Piracicaba Dental School, University of Campinas, Piracicaba, Sao Paulo, Brazil
}

\section{ABSTRACT}

Purpose: The objective of the present study was to evaluate the prevalence of dental implants positioning errors and their associations with adjacent structures and anatomical variations by means of cone-beam computed tomography (CBCT).

Materials and Methods: CBCT images of 207 patients (584 dental implants) were evaluated by 2 oral radiologists. The distance between the implant and the adjacent teeth/implants was measured and classified as adequate $(\geq 1.5 \mathrm{~mm}$ and $\geq 3 \mathrm{~mm}$, respectively) or inadequate. The presence of thread exposure, cortical perforation, implant dehiscence, implant penetration into adjacent structures, and anatomical variations was also recorded. The incisor canal diameter and the depth of the concavity of the submandibular fossa were measured in order to evaluate their correlations with the frequency of implant penetration in these structures. Descriptive analyses, the Fisher exact test, and Spearman correlation analysis were performed $(\alpha=0.05)$.

Results: The overall prevalence of positioning errors was $82.9 \%$. The most common error was the inadequate distance between the implant and the adjacent teeth/implants. The presence of anatomical variations did not significantly influence the overall prevalence of errors $(P>0.05)$. There was a positive correlation between the diameter of the incisor canal and the frequency of implant penetration in this structure $(\mathrm{r}=0.232, P<0.05)$.

Conclusion: There was a high prevalence of dental implant positioning errors, and positioning errors were not associated with the presence of anatomical variations. Professionals should be aware of the space available for implant placement during the preoperative planning stage. (Imaging Sci Dent 2020; 50: 281-90)

KEY WORDS: Anatomic Variation; Cone-Beam Computed Tomography; Dental Implant

\section{Introduction}

Dental implants are an effective alternative to oral rehabilitation and contribute to patients' quality of life. Nevertheless, the implant placement procedure is not exempt from technical errors, which may occur as a result of failures in either surgical planning or the precise identifica-

This study was financed in part by the National Research Council - Brazil (CNPq). Received April 16, 2020; Revised June 26, 2020; Accepted July 21, 2020

*Correspondence to : Dr. Eduarda Helena Leandro Nascimento

Department of Clinical and Preventive Dentistry, Universidade Federal de Pernambuco, Av. Prof. Artur de Sá, 329-481, Cidade Universitária, Recife - PE, Zip code: 52171-011, Brazil

Tel) 55-81-2126-8344, E-mail) Eduarda.hln@gmail.com tion of anatomical structures. ${ }^{1-3}$ Technical errors include an inadequate distance between the implant and adjacent structures, perforation of cortical plates, and penetration into anatomical landmarks. These errors can lead to clinical complications such as failed osseointegration, bone defects, bleeding, neurosensory disorders, and unnecessary additional surgical procedures. Consequently, implant failure (either early or late) has a high likelihood of occurrence. ${ }^{2}$

Cone-beam computed tomography (CBCT) is the most widely recommended imaging modality for implant planning since it allows precise 3-dimensional measurements 
of the implant bone site and accurate identification of anatomical structures and their variations. ${ }^{1}$ For postoperative evaluations, however, the use of CBCT is limited by the production of beam-hardening artifacts and the relatively high radiation dose. ${ }^{4}$ Even so, CBCT is a useful method for evaluating implant positioning errors and is indicated when patients have clinical symptoms such as pain and implant mobility, as it provides additional information when compared to 2-dimensional images. ${ }^{1,3}$ It is also important to consider that technical errors may be related to complications that do not cause immediate symptoms. Thus, the evaluation of implants in CBCT exams acquired for other reasons could assist in the early detection of these errors in a pre-symptomatic stage.

Knowing the prevalence of different types of implant positioning errors and where they most often occur is essential to alert dental practitioners of the importance of proper surgical planning. To date, there are few studies on this subject using a representative sample of CBCT scans. ${ }^{5}$ Moreover, to the authors' best knowledge, it has not been investigated whether the presence of anatomical variations increases the frequency of these errors. Therefore, the purpose of this study was to evaluate the prevalence of dental implant positioning errors and their associations with adjacent structures and anatomical variations by means of CBCT images.

\section{Materials and Methods}

\section{Study sample}

This study was carried out after receiving approval from the local Ethics Committee (protocol \#66784617.9.0000. 5208). The initial sample was composed of 851 consecutive patients referred to an oral radiology center by several professionals for CBCT imaging of the jaws (for a variety of clinical reasons). The inclusion criterion was exams of patients who had at least 1 dental implant. CBCT exams with low technical quality or with a partial image of the implant and adjacent anatomical structures were excluded. After applying the criteria, the final sample was composed of CBCT exams of 207 patients ( 127 women and 80 men aged from 21 to 85 years; mean, $58.1 \pm 12.9$ years), with a total of 584 dental implants (mean, 2.8 implants per patient).

All images were obtained using an i-CAT Next Generation CBCT unit (Imaging Sciences International, Hatfield, $\mathrm{PA}, \mathrm{USA})$. The acquisition parameters $(\mathrm{kVp}, \mathrm{mA}$, and field of view) were selected according to each patient's characteristics and treatment needs, but the voxel size was set at $0.2 \mathrm{~mm}$. Subsequently, the images were exported in Digital Imaging and Communications in Medicine format and evaluated using the OnDemand 3D software (Cybermed Inc., Seoul, Korea).

\section{Image evaluation}

Multiplanar reconstructions (axial, coronal, sagittal, and cross-sectional) were evaluated simultaneously by 2 examiners, an oral radiologist and a dental practitioner with at least 3 years of experience in evaluating CBCT images, by consensus. The evaluations were performed under low-light conditions using a 19-inch LCD monitor (Itautec, São Paulo, Brazil). Brightness, contrast, and zoom tools could be used at the discretion of the examiners.

Patients' data and the region of insertion of each dental implant were recorded. The bilateral absence of teeth or implants adjacent to the evaluated implant was recorded as "absence of adjacent tooth/implant." When adjacent teeth and/or implants were present, the smallest linear distance between them and the evaluated implant was measured. If there was $<1.5 \mathrm{~mm}$ between the tooth and implant and/or $<3 \mathrm{~mm}$ between 2 implants, this relationship was classified as inadequate. ${ }^{6}$ Cases of contact between the implant and the adjacent tooth/implant were recorded as "tooth/implant injury" (Fig. 1).

Thread exposure was evaluated by measuring the mesial, distal, buccal, and lingual bone levels related to the dental implants. The mean value was calculated (in millimeters), and a bone level of up to $2 \mathrm{~mm}$ between the implant shoulder and the alveolar bone crest, without exposure of the implant threads, was considered to be adequate. Cases with a bone loss greater than $2 \mathrm{~mm}$ and absence of bone walls around the entire implant were recorded as thread exposure (Fig. 2)., ${ }^{2,7}$

Perforation/fenestration of the cortical plates and implant dehiscence were considered present, respectively, when the apex of the implant crossed the cortical plate or when it was not possible to identify the bone surrounding part of the implant body in the cervical third (i.e., when 1 of the cortical plates was absent). ${ }^{8}$ For both implant complications, the involved cortical plate (buccal or lingual) was recorded (Fig. 3).

Cases of implant penetration (at least $1 \mathrm{~mm}$ ) into the following anatomical structures were evaluated: incisive canal, nasal cavity, maxillary sinus, mental foramen, and mandibular canal. The presence of anatomical variations such as anterior extension of the maxillary sinus (anterior to premolars) and the anterior loop of the mandibular ca$\mathrm{nal}^{9}$ was also recorded. Canalis sinuosus, when identified, 

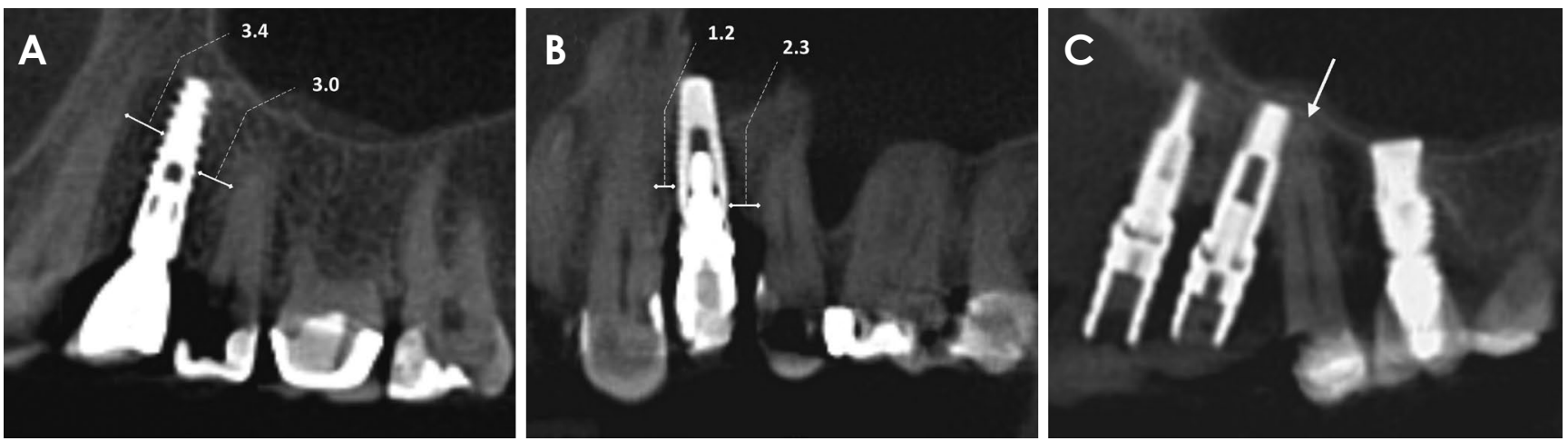

Fig. 1. Sagittal cone-beam computed tomography reconstructions illustrating the relationship between the implant and adjacent tooth. A. Adequate relationship $(>1.5 \mathrm{~mm})$. B. Inadequate relationship $(<1.5 \mathrm{~mm})$. C. Tooth injury (arrow).
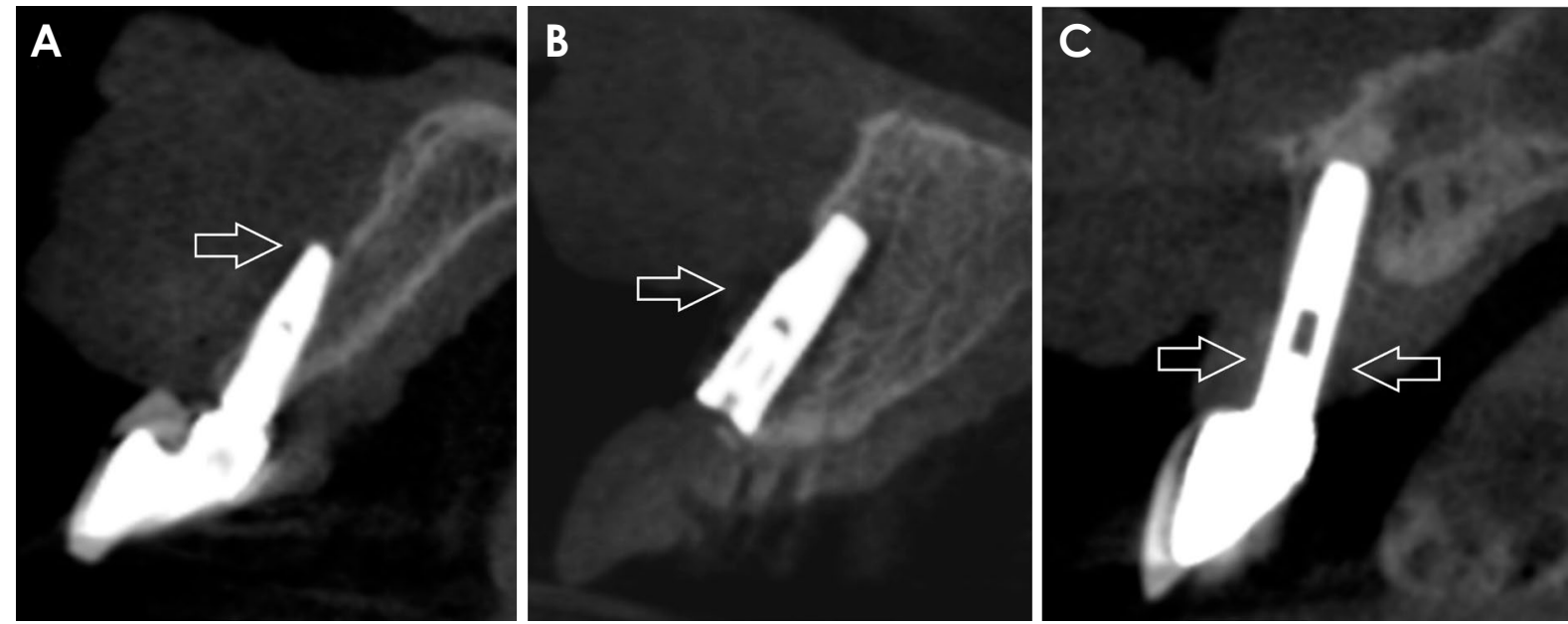

Fig. 2. Cross-sectional cone-beam computed tomography reconstructions show perforation of the buccal cortical plate (A), implant dehiscence related to the buccal cortical plate (B), and thread exposure (C).

was also recorded as an anatomical variation (Fig. 4). ${ }^{10}$

Additional evaluations were performed to identify correlations between the morphology of anatomical structures and the frequency of penetration of the implant into those structures. Thus, for implants located in the region of the maxillary central incisors, the diameter of the incisive canal was measured in its greatest mesiodistal dimension. Likewise, when evaluating implants located in the region of the mandibular molars, the depth of the concavity of the submandibular fossa was measured by tracing a perpendicular line from the deepest area of the submandibular fossa to a reference line tangent to the most prominent upper and lower points of the lingual concavity on cross-sectional CBCT images. ${ }^{11}$

\section{Statistical analysis}

Statistical analyses were performed using SPSS ver- sion 23.0 (IBM Corp., Armonk, NY, USA). Data were grouped according to the region of implant insertion and a descriptive analysis with the distribution of absolute and relative frequencies was performed. The Fisher exact test was used to evaluate the association between the presence of anatomical variations and the prevalence of implant complications. The Spearman correlation test was used to assess the influence of the depth of concavity of the submandibular fossa and the incisive canal diameter on the frequency of implant perforation in these respective structures. The significance level was set to $P<0.05$.

\section{Results}

The general prevalence of implant positioning errors and the distribution of individual and co-occurring errors according to the location of dental implants are shown in 

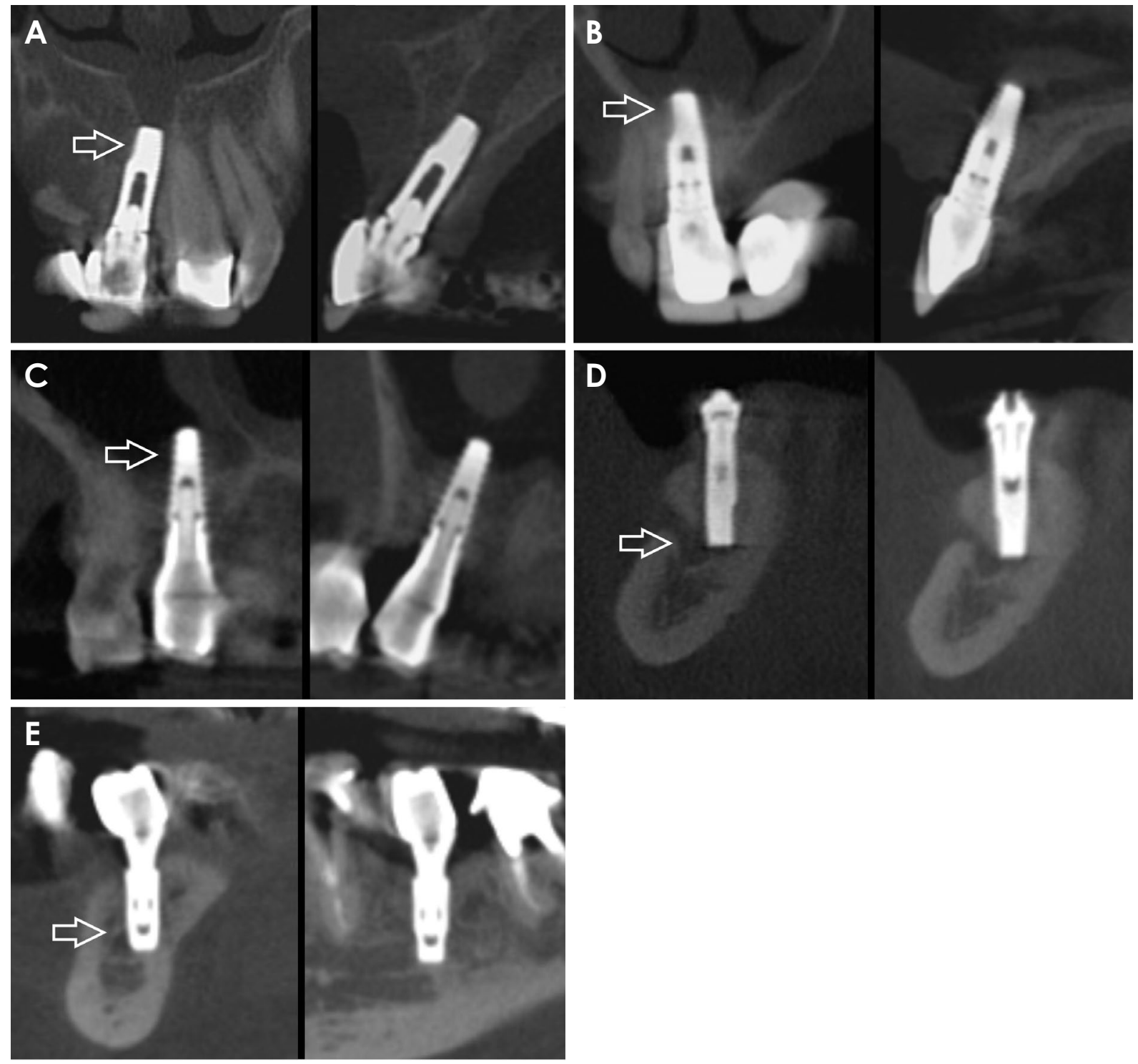

Fig. 3. Cone-beam computed tomography reconstructions show (arrows) implant penetration into the incisive canal (A), nasal fossa (B), maxillary sinus (C), mental foramen (D), and mandibular canal (E).

Table 1. There was a high general prevalence of errors in both the maxilla (85.7\%) and the mandible (75.6\%), resulting in an average error of $82.9 \%$ in the study sample. The highest and the lowest error rates were found in the regions of the maxillary central incisors (94.4\%) and maxillary molars $(69.2 \%)$, respectively. The most frequent errors that occurred individually were inadequate distance/ injury for the maxillary teeth $(21.9 \%)$ and thread exposure for the mandibular teeth (22\%). Co-occurrence of 2 or more positioning errors was observed frequently, with rates ranging from $14.3 \%$ to $55.6 \%$ of cases depending on the region (Fig. 5). The most common combinations were inadequate distance/injury with implant dehiscence and inadequate distance/injury with penetration into anatomical structures; these combinations represented 27.4\% (55) and $22.4 \%$ (45) of all cases of multiple positioning errors, respectively.

Table 2 details the variables of the study and the distribution of types of positioning errors according to the location of dental implants. For maxillary implants, inadequate distance to the adjacent tooth/implant was the most frequent error in all regions (59.7\% to $68.1 \%$ ), except for the molar region, where the most common error was penetration into the maxillary sinus (53.8\%). In contrast, for mandibular implants, the most frequent errors were thread exposure and inadequate distance to the adjacent teeth/ 

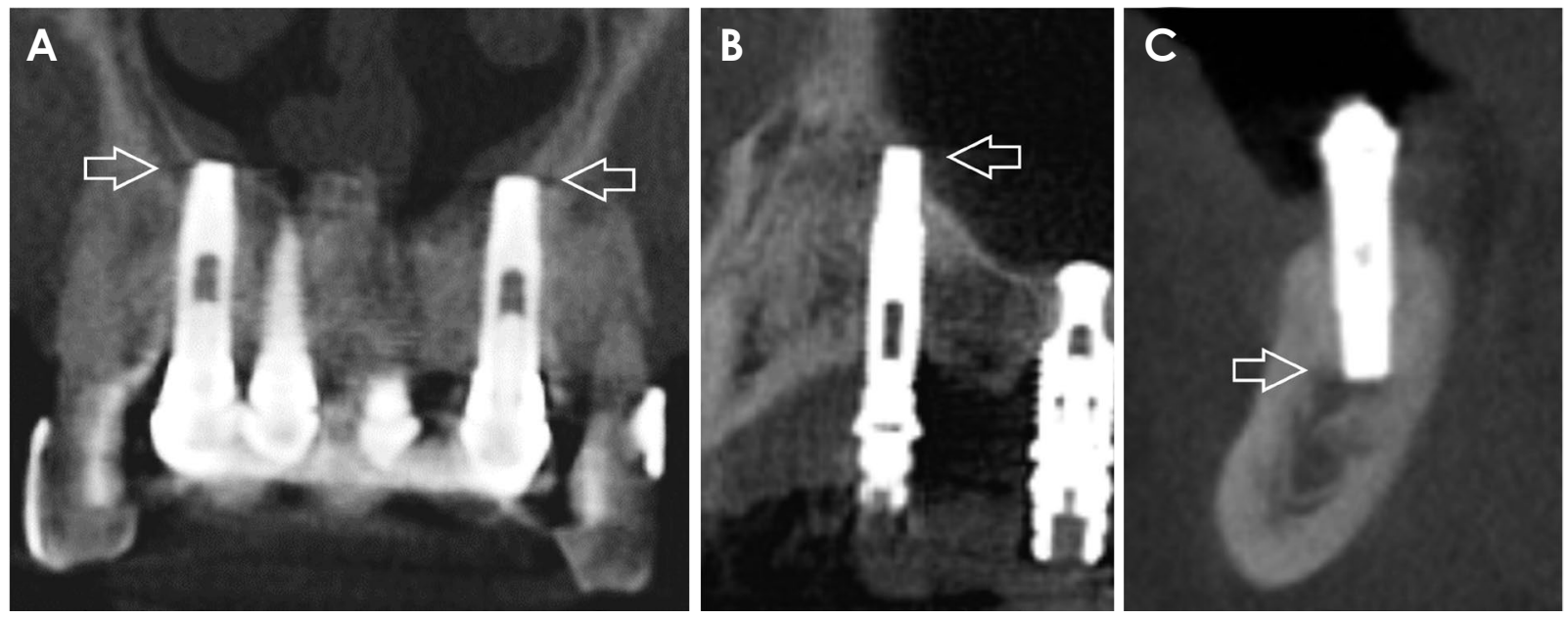

Fig. 4. Coronal and cross-sectional cone-beam computed tomography reconstructions reveal implants penetrating some anatomical variations (arrows). A. Canalis sinuosus. B. Anterior extension of the maxillary sinus. C. Anterior loop of the mandibular canal.

Table 1. General prevalence of implant positioning errors and the distribution of individual and co-occurring errors according to the location of dental implants (number and percentage)

\begin{tabular}{|c|c|c|c|c|c|c|c|c|}
\hline Implant region & $\begin{array}{l}\text { No } \\
\text { positioning } \\
\text { errors }\end{array}$ & $\begin{array}{l}\text { Inadequate } \\
\text { distance/ } \\
\text { injury }\end{array}$ & $\begin{array}{l}\text { Thread } \\
\text { exposure }\end{array}$ & $\begin{array}{l}\text { Cortical } \\
\text { perforation }\end{array}$ & $\begin{array}{c}\text { Implant } \\
\text { dehiscence }\end{array}$ & $\begin{array}{c}\text { Penetration } \\
\text { into anatomical } \\
\text { structures* }\end{array}$ & $\begin{array}{l}\text { Co-occurrence of } \\
\text { positioning } \\
\text { errors } * *\end{array}$ & Total \\
\hline \multicolumn{9}{|l|}{ Maxilla } \\
\hline Central incisor $(\mathrm{n}=72)$ & $4(5.6)$ & $15(20.8)$ & $3(4.2)$ & $1(1.4)$ & $7(9.7)$ & $2(2.8)$ & $40(55.6)$ & $68(94.4)$ \\
\hline Lateral incisor $(n=64)$ & $4(6.2)$ & $18(28.1)$ & $3(4.7)$ & $1(1.6)$ & $7(10.9)$ & $3(4.7)$ & $28(43.8)$ & $60(93.8)$ \\
\hline Canine $(n=47)$ & $9(19.1)$ & $6(12.8)$ & $7(14.9)$ & $1(2.1)$ & $13(27.7)$ & $4(8.5)$ & 7 (14.9) & $38(80.9)$ \\
\hline $\operatorname{Premolar}(\mathrm{n}=159)$ & $19(11.9)$ & $41(25.8)$ & $5(3.1)$ & $3(1.9)$ & $15(9.4)$ & $8(5.0)$ & $68(42.8)$ & $140(88.1)$ \\
\hline $\operatorname{Molar}(\mathrm{n}=78)$ & $24(30.8)$ & $12(15.3)$ & $3(3.8)$ & - & $4(5.1)$ & $11(14.1)$ & $24(30.8)$ & $54(69.2)$ \\
\hline Total $(n=420)$ & $60(14.3)$ & $92(21.9)$ & $21(5.0)$ & $6(1.4)$ & $46(11.0)$ & $28(6.7)$ & $167(40.0)$ & $360(85.7)$ \\
\hline \multicolumn{9}{|l|}{ Mandible } \\
\hline Anterior teeth $(\mathrm{n}=21)$ & $3(14.3)$ & - & $8(38.1)$ & - & $2(9.5)$ & - & $8(38.1)$ & $18(85.7)$ \\
\hline Premolars $(\mathrm{n}=52)$ & $10(19.2)$ & $10(19.2)$ & $8(15.4)$ & - & $11(21.2)$ & - & $13(25.0)$ & $42(80.8)$ \\
\hline Molars $(n=91)$ & $27(29.7)$ & $8(8.8)$ & $20(22.0)$ & $4(4.4)$ & $17(18.7)$ & $2(2.2)$ & $13(14.3)$ & $64(70.3)$ \\
\hline $\operatorname{Total}(n=164)$ & $40(24.4)$ & $18(11.0)$ & $36(22.0)$ & $4(2.4)$ & $30(18.3)$ & $2(1.2)$ & $34(20.7)$ & $124(75.6)$ \\
\hline Total $(n=584)$ & $100(17.1)$ & $110(18.8)$ & $57(9.8)$ & $10(1.7)$ & $76(13)$ & $30(5.1)$ & $201(34.4)$ & $484(82.9)$ \\
\hline
\end{tabular}

*: penetration into at least 1 anatomical structure, **: co-occurrence of 2 or more positioning errors. Mandibular anterior teeth are grouped because there were few cases of implants located in these regions.

implants in the anterior region (each present in $66.7 \%$ of cases), implant dehiscence in the premolar region $(40.4 \%$, considering buccal and lingual cases) and thread exposure in the molar teeth $(29.7 \%)$.

In general, the mean distance from implants to adjacent teeth/implants increased as the region of implant insertion became more posterior, in both the maxilla and the mandible (Table 2). Regarding the penetration into anatomical structures, there was a high prevalence of penetration into the maxillary sinus (34.2\%, 81 of 237 implants), followed by implants reaching the incisive canal $(12.5 \%, 9$ of 72 implants), nasal cavity $(6.4 \%, 27$ of 420 implants), mandibular canal (5.6\%, 8 of 143 implants) and mental foramen (4.1\%, 3 of 73 implants). The presence of anatomical variations was also related to high penetration rates into the anterior extension of the maxillary sinus (17.7\%, 3 of 17 cases), canalis sinuosus (12.3\%, 13 of 106 cases), and the anterior loop of the mandibular canal $(11.8 \%, 4$ of 34 

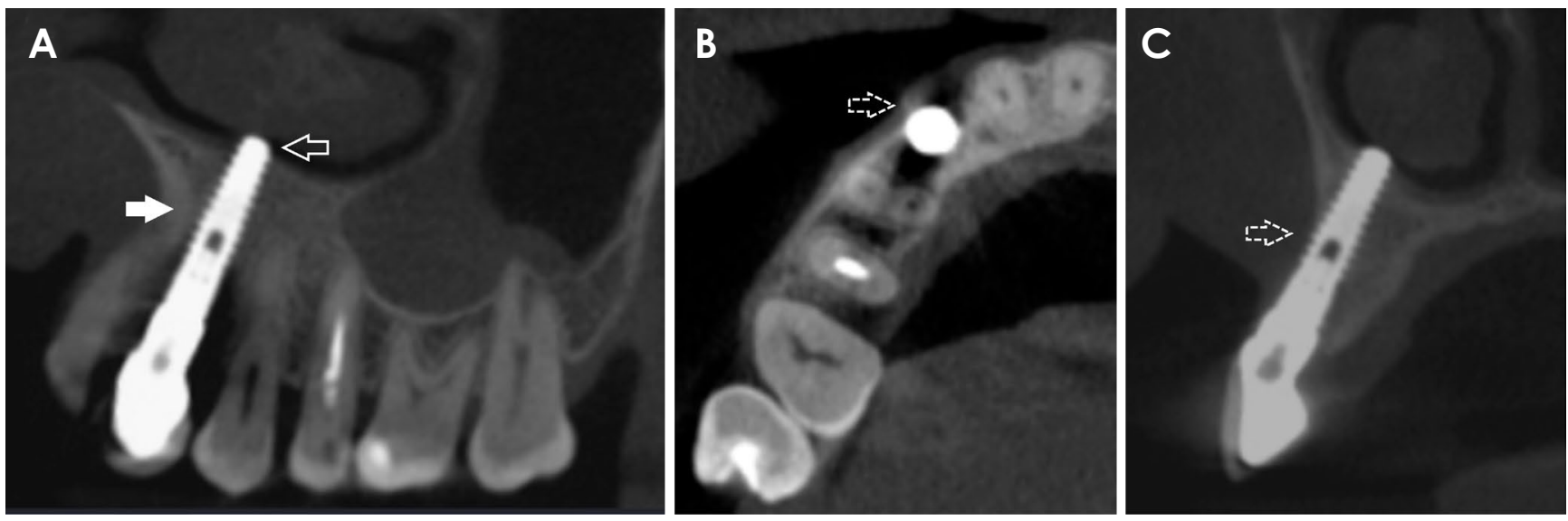

Fig. 5. Sagittal (A), axial (B), and cross-sectional (C) cone-beam computed tomographic images from a patient show multiple implant positioning errors: an inadequate distance between the implant and adjacent tooth (full arrow), penetration into an anatomical structure (the nasal cavity; empty arrow), and implant dehiscence (dotted arrow).

cases: 2 cases in the canine region and 32 cases in the premolar region) (Table 2).

According to the Fisher exact test, the presence of anatomical variations did not significantly influence the overall prevalence of implant positioning errors $(P>0.05)$. Spearman correlation analysis showed a positive and significant correlation between the incisive canal diameter and the frequency of implant penetration into this structure $(\mathrm{r}=0.232$, slight correlation; $P<0.05)$. On the other hand, the depth of the concavity of the submandibular fossa did not influence the frequency of implant perforation on the lingual cortical plate in the posterior region of the mandible $(P=0.923)$.

\section{Discussion}

The present study showed a high overall prevalence of dental implant positioning errors (82.9\%), and an inadequate distance between implant and adjacent teeth/ implants was the most common error. Although previous studies have also investigated this issue using $\mathrm{CBCT}^{2,5,7}$ to the authors' best knowledge, none have performed an analysis of the prevalence of all possible implant positioning errors and their combinations. Furthermore, no previous study has investigated whether the presence of anatomical variations at the implant site could increase the occurrence of errors. It is known that neglecting anatomical landmarks in the preoperative evaluation can cause harm to the patient. However, surprisingly, it was observed that the presence of anatomical variations did not significantly influence the findings of the present study.
The overall and specific prevalence rates of implant positioning errors observed here were much higher than those reported in the literature $(6.89 \%$ to $37.5 \%),{ }^{2,5,7}$ and it is also noteworthy that multiple errors were present in many cases $(34.4 \%)$. This discrepancy can be explained by the different criteria used in various studies for evaluating errors. While some studies considered only some types of positioning errors in their analyses, ${ }^{2,5,7}$ others did not distribute error types according to different regions of implant insertion (instead making a percentage calculation over the total sample). ${ }^{2}$ This may mask the actual prevalence of errors and cause misinterpretation of possible location-related complications. ${ }^{12}$ In addition, possible differences in sample size, study population, and analysis methods should be considered. In the study conducted by Gaêta-Araujo et al., for example, the authors did not present the general prevalence of errors, but instead reported independent prevalence rates for perforations into anatomical structures (33.3\%) and inadequate distance between the implant and the adjacent tooth/implant (18.2\%). Furthermore, this last value may have been lower than that reported in the present study because those authors considered only cases with a distance $<1 \mathrm{~mm}$ as inadequate, whereas the cutoff used in the present study was $<1.5 \mathrm{~mm}$.

In general, the average implant distance to the adjacent tooth/implant increased in more posterior regions. The average values were below the recommended distance ${ }^{6,13}$ in the maxillary lateral incisor region (considering the implant-tooth relationship) and in the region of the central incisors to maxillary premolars (considering the implant-implant relationship). Such positioning errors can 


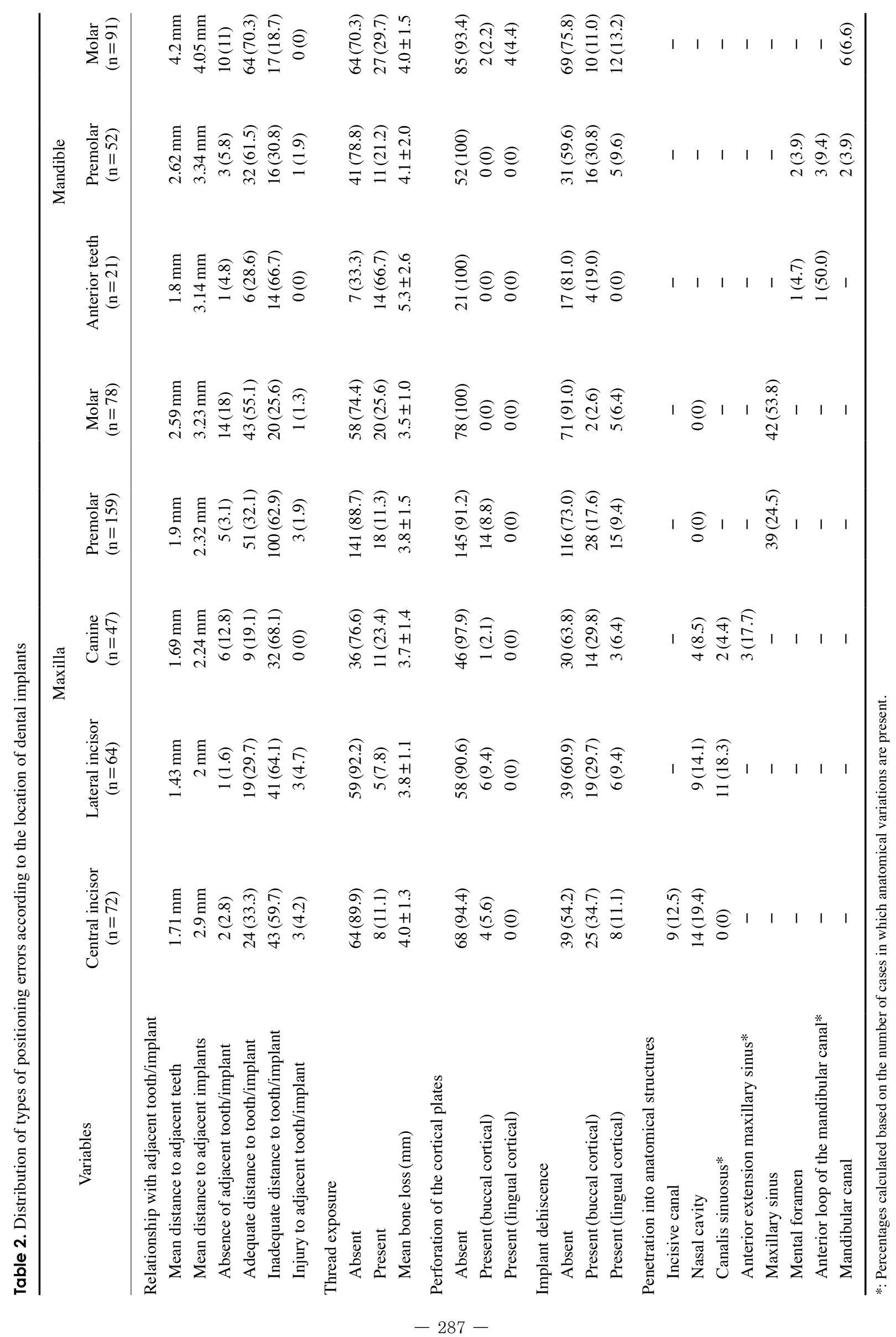


lead to hypersensitivity and discomfort while eating, ${ }^{14}$ as well as inducing implant thread exposure or horizontal bone loss, ${ }^{5,15}$ thereby influencing the aesthetic outcome of oral rehabilitation. In this context, platform-switching implants can be used as an alternative for rehabilitation if limited space is available for implant placement. This is because, when using platform-switching implants, the distance between the implant and the adjacent teeth could be as close as $1 \mathrm{~mm}$ without interfering negatively with the crestal bone level, ${ }^{16}$ which could reduce the incidence of inadequate distance errors. When in contact with an adjacent tooth, the implant may lead to its resorption. This was more frequent in the maxillary anterior region, as previously reported. ${ }^{5,7}$ These results can be attributed to the mesiodistal space available for the accommodation of dental implants, which in many cases is insufficient in this region. ${ }^{13}$

Wide variation was found in the prevalence of implant thread exposure depending on the region evaluated $(7.8 \%$ to $66.7 \%$ - in the anterior mandible). Silva et al. ${ }^{7}$ found that thread exposure was the most common type of positioning error (37.5\%), occurring most commonly in the anterior maxilla. Thread exposure can be influenced by the time after implant insertion, and bone loss up to 2 $\mathrm{mm}$ in the first year of implant placement is expected and acceptable. ${ }^{17}$ As this was a retrospective cross-sectional study based only on image evaluations, this clinical information was not available, making it impossible to consider whether thread exposure occurred due to bone loss or insufficient insertion. In contrast, when evaluating implant thread exposure on immediate postoperative CBCT scans, Clark et al. ${ }^{2}$ found only 4 cases (out of 160 implants with some error), classifying them as implants with insufficient insertion. Future studies with clinical and longitudinal information would certainly contribute even more to knowledge on the prevalence of bone loss and thread exposure over time.

Cortical perforation and implant dehiscence are both peri-implant bone defects usually caused by mispositioning of implants in the buccolingual direction of the alveolar ridge. In cases of implant dehiscence, the apposition of fibrous scar tissue may be found, which hinders bone remodeling in the region and may lead to implant mobility, in addition to aesthetic damage to the gingival tissue, especially when it involves the buccal cortical plate. ${ }^{2}$ In contrast, perforation is mainly worrisome when it occurs in the mandibular lingual region due to the presence of the submandibular and sublingual arteries, which pose a risk for intraoral floor hemorrhage. ${ }^{3}$ In the present study, the depth of the concavity of the submandibular fossa did not influence the prevalence of lingual cortical perforation in the posterior region of the mandible. These findings contradict those of previous studies that evaluated fossa concavity and, through virtual implant placement planning, suggested that deep concavity of the fossa may be related to a higher risk of perforation. ${ }^{11,18-20}$

Penetration into anatomical structures was also highly prevalent in the sample, especially in the maxilla. According to the Spearman test, the larger the diameter of the incisor canal, the greater the occurrence of perforation into it. Injuries to this structure may cause damage to the nasopalatine nerve and, consequently, sensory loss in the region. ${ }^{21}$ Evidence shows that penetration of implants into the maxillary sinus or nasal fossa can lead to mucosal inflammation and the development of postoperative rhinitis or sinusitis, ${ }^{22}$ although some authors have argued that there may be no major complications. ${ }^{2}$ In the mandible, the frequency of implant penetration into the mandibular canal and the mental foramen was similar (3.9\% to 6.6\%). In such cases, the possible presence of neurosensorial disorders should be considered..$^{23}$ The major cause of mandibular canal injury is surgical planning with conventional radiographs only, so these findings support the importance of CBCT in the preoperative stage.

Anatomical variations are usually unexpected. Therefore, the initial hypothesis in this study was that their presence would significantly increase the prevalence of implant positioning errors. This association was most likely not significant due to the large number of errors in the overall sample. In addition to the anatomical variations evaluated, the presence of mandibular canal bifurcations and accessory foramina was also taken into consideration. However, as only the dental implant regions were assessed, these variations were not found in the sample of this study.

According to guidelines for CBCT clinical use, this imaging modality should be used postoperatively only when there is evidence of implant complications. ${ }^{1,324}$ However, implants present in CBCT images performed for any reason should also be carefully evaluated. Furthermore, it is known that acquisition parameters may influence the diagnostic performance of CBCT. ${ }^{25,26}$ The smaller the voxel size, for example, the better the image quality and the lower the occurrence of the partial volume effect, but also the higher the radiation dose required..$^{12,24,25}$ The parameters used in this study are consistent with those commonly used for full arch acquisitions. Moreover, even with inherent drawbacks such as the production of artifacts 
by implants, which can mask the visualization of neurovascular bundles and peri-implant bone defects, ${ }^{3}$ CBCT has shown clinically acceptable performance for this purpose. $^{25}$

Positioning errors cannot be considered as equivalent to implant failure. Failure occurs when there is implant loss or when implant replacement is required ${ }^{26}$ thus, implant failure involves several clinical factors that were not available in the present study. According to the literature, failure occurs in about $2.9 \%$ to $7.2 \%$ of cases $^{27,28}$ and may be influenced by factors such as patient age, the presence of a prosthesis, and the type of implant. Improper angulation of dental implants may also contribute to peri-implant bone loss, as well as leading to unwanted aesthetic results, which would result in implant failure. Given the absence of clinical data, this factor was likewise not evaluated in the present study. Therefore, future studies in other populations, correlating imaging findings with clinical data, may further contribute to knowledge in this field of research.

In conclusion, a high prevalence of dental implant positioning errors was found, and these errors were not associated with the presence of anatomical variations. Even so, any anatomical variation should be considered when planning dental implant surgery so that errors involving these structures can be avoided. Dental practitioners should also be aware of the space available for implant placement during preoperative planning, since inadequate distance between implants and adjacent teeth/implants was the most common error.

\section{Conflicts of Interest: None}

\section{References}

1. Tyndall DA, Price JB, Tetradis S, Ganz SD, Hildebolt C, Scarfe WC, et al. Position statement of the American Academy of Oral and Maxillofacial Radiology on selection criteria for the use of radiology in dental implantology with emphasis on cone beam computed tomography. Oral Surg Oral Med Oral Pathol Oral Radiol 2012; 113: 817-26.

2. Clark D, Barbu H, Lorean A, Mijiritsky E, Levin L. Incidental findings of implant complications on postimplantation CBCTs: a cross-sectional study. Clin Implant Dent Relat Res 2017; 19: 776-82.

3. Jacobs R, Salmon B, Codari M, Hassan B, Bornstein MM. Cone beam computed tomography in implant dentistry: recommendations for clinical use. BMC Oral Health 2018; 18: 88.

4. Fontenele RC, Nascimento EH, Vasconcelos TV, Noujeim M, Freitas DQ. Magnitude of cone beam CT image artifacts related to zirconium and titanium implants: impact on image quality. Dentomaxillofac Radiol 2018; 47: 2018021.

5. Gaêta-Araujo H, Oliveira-Santos N, Mancini AX, Oliveira ML, Oliveira-Santos C. Retrospective assessment of dental implant-related perforations of relevant anatomical structures and inadequate spacing between implants/teeth using conebeam computed tomography. Clin Oral Investig 2020; 24 : 3281-8.

6. Tarnow DP, Cho SC, Wallace SS. The effect of inter-implant distance on the height of inter-implant bone crest. J Periodontol 2000; 71: 546-9.

7. Silva JA, de Alencar AH, da Rocha SS, Lopes LG, Estrela C. Three-dimensional image contribution for evaluation of operative procedural errors in endodontic therapy and dental implants. Braz Dent J 2012; 23: 127-34.

8. de-Azevedo-Vaz SL, Vasconcelos Kde F, Neves FS, Melo SL, Campos PS, Haiter-Neto F. Detection of periimplant fenestration and dehiscence with the use of two scan modes and the smallest voxel sizes of a cone-beam computed tomography device. Oral Surg Oral Med Oral Pathol Oral Radiol 2013; 115: 121-7.

9. do Nascimento EH, Dos Anjos Pontual ML, Dos Anjos Pontual A, da Cruz Perez DE, Figueiroa JN, Frazão MA, et al. Assessment of the anterior loop of the mandibular canal: a study using cone-beam computed tomography. Imaging Sci Dent 2016; 46: 69-75.

10. de Oliveira-Santos C, Rubira-Bullen IR, Monteiro SA, León JE, Jacobs R. Neurovascular anatomical variations in the anterior palate observed on CBCT images. Clin Oral Implants Res 2013; 24: 1044-8.

11. Parnia F, Fard EM, Mahboub F, Hafezeqoran A, Gavgani FE. Tomographic volume evaluation of submandibular fossa in patients requiring dental implants. Oral Surg Oral Med Oral Pathol Oral Radiol Endod 2010; 109: e32-6.

12. Nascimento EH, Oliveira ML, Freitas DQ. Incidental findings of implant complications on postimplantation CBCTs: a cross-sectional study - methodological issues. Clin Implant Dent Relat Res 2019; 21: 11-2.

13. Wilson JP, Johnson TM. Frequency of adequate mesiodistal space and faciolingual alveolar width for implant placement at anterior tooth positions. J Am Dent Assoc 2019; 150: 779-87.

14. Camargo IB, Van Sickels JE. Surgical complications after implant placement. Dent Clin North Am 2015; 59: 57-72.

15. Scarano A, Assenza B, Piattelli M, Thams U, San Roman F, Favero GA, et al. Interimplant distance and crestal bone resorption: a histologic study in the canine mandible. Clin Implant Dent Relat Res 2004; 6: 150-6.

16. Vela X, Méndez V, Rodríguez X, Segalá M, Tarnow DP. Crestal bone changes on platform-switched implants and adjacent teeth when the tooth-implant distance is less than 1.5 mm. Int J Periodontics Restorative Dent 2012; 32: 149-55.

17. Gupta S, Sabharwal R, Nazeer J, Taneja L, Choudhury BK, Sahu S. Platform switching technique and crestal bone loss around the dental implants: a systematic review. Ann Afr Med 2019; 18: 1-6.

18. Chan HL, Benavides E, Yeh CY, Fu JH, Rudek IE, Wang HL. Risk assessment of lingual plate perforation in posterior mandibular region: a virtual implant placement study using cone- 
beam computed tomography. J Periodontol 2011; 82: 129-35.

19. Huang RY, Cochran DL, Cheng WC, Lin MH, Fan WH, Sung $\mathrm{CE}$, et al. Risk of lingual plate perforation for virtual immediate implant placement in the posterior mandible: a computer simulation study. J Am Dent Assoc 2015; 146: 735-42.

20. Lin MH, Mau LP, Cochran DL, Shieh YS, Huang PH, Huang RY. Risk assessment of inferior alveolar nerve injury for immediate implant placement in the posterior mandible: a virtual implant placement study. J Dent 2014; 42: 263-70.

21. de Mello JS, Faot F, Correa G, Chagas Júnior OL. Success rate and complications associated with dental implants in the incisive canal region: a systematic review. Int J Oral Maxillofac Surg 2017; 46: 1584-91.

22. Mahale KM, Yeshwante BJ, Baig N, Darakh PG. Iatrogenic complications of implants surgery. J Dent Implant 2013; 3: 157-9.

23. Juodzbalys G, Wang HL, Sabalys G. Injury of the inferior alveolar nerve during implant placement: a literature review. J Oral Maxillofac Res 2011; 2: e1.
24. Bornstein MM, Scarfe WC, Vaughn VM, Jacobs R. Cone beam computed tomography in implant dentistry: a systematic review focusing on guidelines, indications, and radiation dose risks. Int J Oral Maxillofac Implants 2014; 29 Suppl: 55-77.

25. Bohner LO, Mukai E, Oderich E, Porporatti AL, Pacheco-Pereira $\mathrm{C}$, Tortamano $\mathrm{P}$, et al. Comparative analysis of imaging techniques for diagnostic accuracy of peri-implant bone defects: a meta-analysis. Oral Surg Oral Med Oral Pathol Oral Radiol 2017; 124: 432-40.e5.

26. Oztel M, Bilski WM, Bilski A. Risk factors associated with dental implant failure: a study of 302 implants placed in a regional center. J Contemp Dent Pract 2017; 18: 705-9.

27. Hickin MP, Shariff JA, Jennette PJ, Finkelstein J, Papapanou PN. Incidence and determinants of dental implant failure: a review of electronic health records in a U.S. dental school. J Dent Educ 2017; 81: 1233-42.

28. Jemt T. Implant failures and age at the time of surgery: a retrospective study on implant treatment in 2915 partially edentulous jaws. Clin Implant Dent Relat Res 2019; 21: 686-92. 